\title{
Clinical Outcomes and Medication Adherence in Acute Coronary Syndrome Patients With and Without Type 2 Diabetes Mellitus: A Longitudinal Analysis 2006-2011
}

\author{
Mark J. Cziraky, PharmD, CLS; Vanessa S. Reddy, MS; Rakesh Luthra, MS; Yaping Xu, MD, MPH;
} Kenneth Wilhelm, MD; Thomas P. Power, MD; and Maxine D. Fisher, PhD

\begin{abstract}
BACKGROUND: The presence of type 2 diabetes mellitus magnifies the risks associated with acute coronary syndrome (ACS), increasing the risk of recurrent cardiovascular events (CVEs) and doubling the risk of death. Managing cardiovascular risk factors has little effect on lowering the mortality risk in patients with type 2 diabetes.
\end{abstract}

OBJECTIVE: To evaluate the relationship between type 2 diabetes mellitus and subsequent CVEs and medication adherence following ACS hospitalization.

METHODS: Patients with ACS were identified using ICD-9-CM codes for acute myocardial infarction or unstable angina. The risk of subsequent CVEs was assessed at 1 and 3 years after the index ACS event based on type 2 diabetes status, adjusting for baseline demographic characteristics, comorbidities, medication use, and index ACS characteristics.

RESULTS: Of 140,903 patients with ACS (mean age 66.8 years, $58.6 \%$ male), $27.4 \%$ had type 2 diabetes. During follow-up, $22.0 \%$ had subsequent CVEs (26.2\% type 2 diabetes, $19.0 \%$ nondiabetes). After adjusting for other covariates, type 2 diabetes was associated with increased risk of subsequent CVEs by $9.7 \%$ at 1 year and $10.2 \%$ at 3 years (both $P<0.001$ ). Most patients were not revascularized at first recurrence after index ACS discharge (79.2\% type 2 diabetes, $77.5 \%$ nondiabetes). Patients with type 2 diabetes had statistically significant higher adherence rates for antiplatelet agents at 1 year and antihypertensives at 1 and 3 years versus nondiabetes patients. Persistence was higher in the type 2 diabetes group for antihypertensives and in the nondiabetes group for antiplatelet agents and statins.

CONCLUSIONS: This analysis demonstrates that patients with type 2 diabetes have a higher risk of subsequent CVEs following an initial event versus those without diabetes, despite evidence of higher treatment persistence for certain medications. Adherence rates remained suboptimal, suggesting a continuing need for patient education.

J Manag Care Spec Pharm. 2015;21(6):470-77

Copyright $\odot 2015$, Academy of Managed Care Pharmacy. All rights reserved.

\section{What is already known about this subject}

Patients with type 2 diabetes have more extensive cardiovascular disease (CVD) and a higher prevalence of hypertension, heart failure, peripheral vascular disease, and neuropathy.

Type 2 diabetes is an independent risk factor for all types of CVDs, as well as long-term mortality and need for revascularization.
After acute coronary syndrome (ACS), patients with type 2 diabetes remain at high risk for subsequent cardiovascular events (CVEs), despite intensive management with standard-of-care therapies.

\section{What this study adds}

In this real-world analysis of patients who had been hospitalized with an ACS event, patients with type 2 diabetes had a higher rate of subsequent CVEs than those without diabetes.

The majority of patients in the type 2 diabetes and nondiabetes cohorts did not undergo revascularization at the time of the initial ACS event.

In general, adherence rates were not statistically different between patients with and without type 2 diabetes, but persistence was higher among patients with type 2 diabetes.

Our findings show that there is opportunity to increase the use of, and adherence with, guideline-recommended treatment by encouraging and monitoring treatment of patients with type 2 diabetes following ACS

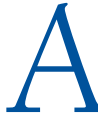
cute coronary syndrome (ACS) contributes to an estimated 1.3 million hospitalizations in the United States annually.,2 ACS presents short- and long-term risks, with $20 \%$ of patients having a subsequent major cardiovascular event (CVE) within 3 years of the initial ACS diagnosis, even with successful treatment of that first event. ${ }^{3}$

The risks associated with ACS are magnified in the presence of type 2 diabetes mellitus., ${ }^{4,5}$ Patients with type 2 diabetes not only have more extensive cardiovascular disease (CVD), but they also have a higher prevalence of hypertension, heart failure, peripheral vascular disease, and neuropathy. ${ }^{6}$ Type 2 diabetes is an independent risk factor for all types of CVDs, as well as long-term mortality and need for revascularization. ${ }^{6}$ For patients with type 2 diabetes, the risk of death is approximately double that for those without diabetes; the risk of death specifically from CVD is tripled. ${ }^{7}$ Optimal management of cardiovascular risk factors does not appear to lower mortality risk in patients with type 2 diabetes. ${ }^{7}$

Patients surviving an ACS event are at increased risk of recurrent CVEs, and this risk is further increased in patients 
with type 2 diabetes. ${ }^{8}$ Clinical studies indicate effective management of post-ACS patients with type 2 diabetes requires aggressive multidisciplinary efforts that include revascularization and intensive management of low-density lipoprotein cholesterol, blood pressure, and thrombotic risk. ${ }^{8}$ However, after an ACS event, patients with type 2 diabetes remain at high risk for subsequent CVEs despite intensive management with standard-of-care therapies, ${ }^{9}$ raising the question of whether patients are nonadherent with prescribed treatment regimens or the treatment regimens are less effective in these patients. Additional longitudinal, real-world data are needed to support the evidence base.

Currently, there is limited published literature regarding the relationship between type 2 diabetes and subsequent CVEs, quantifying long-term medication adherence and persistence following ACS. The increasing prevalence of type 2 diabetes drives the need for studies addressing the management of ACS in type 2 diabetes in routine clinical practice. To fill this knowledge gap, the relationship between type 2 diabetes and subsequent CVEs in patients following hospitalization for an ACS event was assessed using administrative claims data representing a real-world cohort. The objective was to evaluate the long-term impact of type 2 diabetes and associated medication management of ACS on outcomes in patients with ACS in a large, commercially insured U.S. population.

\section{Methods}

\section{Study Design and Setting}

This observational cohort study used administrative claims to evaluate longitudinal outcomes and adherence to guidelinerecommended and nonguideline-recommended medications. The HealthCore Integrated Research Environment database was utilized, consisting of an integrated dataset containing medical, pharmacy, and eligibility claims of insured patients from 14 major commercial health plans across the United States.

This retrospective claims analysis was conducted in compliance with state and federal laws, including the Health Insurance Portability and Accountability Act of 1996. All claims data were from a limited dataset with de-identified patient information. No patients were directly involved in the study; therefore, review by an institutional review board was unnecessary.

\section{Selection of Subjects}

Patients were included in the analysis if they were at least aged 18 years, with at least 1 claim for acute myocardial infarction (MI; International Classification of Diseases, Ninth Revision, Clinical Modification [ICD-9-CM] code 410.xx) or unstable angina (UA; ICD-9-CM code 411.1x) during a hospitalization between January 1, 2006, and September 30, 2011 (identification period). The index date was defined as the date of the first hospitalization for ACS during the identification period.
All patients had at least 12 months of continuous health plan eligibility prior to the index date. Patients with a previous diagnosis of acute MI or UA/ACS within 1 year before the index date were excluded.

Patients were included in the type 2 diabetes cohort if they had 2 or more claims for type 2 diabetes at least 30 days apart, or if they had at least 1 claim for type 2 diabetes plus at least 1 claim for oral or injectable antidiabetic medications, or 2 or more claims for oral antidiabetic agents or glucagon-like peptide 1 medications. Patients with type 1 diabetes were excluded. Patients with no evidence of a diabetes diagnosis (type 1 or type 2) were included in the nondiabetes cohort.

\section{Study Endpoints}

Study endpoints included patient demographic characteristics, geographic region, health plan type, baseline comorbidities (cardiovascular and noncardiovascular), baseline medications, index event, and index procedure. Other endpoints included all-cause mortality, hospitalization for UA, resuscitated arrest, revascularization, and other CVEs (including heart failure, peripheral vascular disease, or hemorrhagic stroke). All endpoint measures were compared between the type 2 diabetes and nondiabetes cohorts. Post-index CVE (stroke, MI, or coronary heart disease [CHD]-related mortality) was evaluated by the number and type of events and time to event recurrence. CVE coding was identified as follows: nonfatal MI (ICD-9-CM codes 410.0x-410.8x), nonfatal stroke (ICD-9-CM codes 430.xx, 431, 432.0-432.9, 434.01, 434.11, 434.91), or CHD (ICD-9-CM codes 410.xx, 411.xx, 414.xx, 441.xx, ICD-9-CM Procedure codes 36.0136.09, 36.1x, 36.2x, and Current Procedural Terminology codes 33510-33516, 33517-33545, 33572, 92975, 92980-92981, 9298292984, 92995-92996). CHD-related mortality was defined as death within 7 days following the CHD event. Recurrence was reported by length of follow-up and stratified by diabetes status and whether patients received standard treatment at discharge (i.e., prescription for antiplatelet, anti-ischemic, antihypertensive, or antidyslipidemic medications and other commonly used treatments, such as anticoagulants, plus medications for glycemic control for patients with diabetes).

To measure adherence to medication, postdischarge use of cardiovascular medications was evaluated longitudinally at 1 and 3 years. The medication possession rate (MPR) was calculated for patients who initiated therapy at any time between the index ACS event and 90 days following discharge from that admission. MPR was defined as the percentage of total number of days' supply of the ACS treatment prescription fill divided by the duration of the follow-up period (i.e., 365 and 1,095 days).

Adherence was measured by the MPR at 1 and 3 years and stratified by length of follow-up. The total length of therapy was described as the lengths of follow-up (i.e., patients with at least 1 year of follow-up, those with 2 years of follow-up, and those with 3 years of follow-up). Treatment 
Clinical Outcomes and Medication Adherence in Acute Coronary Syndrome Patients

With and Without Type 2 Diabetes Mellitus: A Longitudinal Analysis 2006-2011

TABLE 1 Baseline Patient Characteristics

\begin{tabular}{|c|c|c|c|c|c|c|c|c|}
\hline & \multirow{2}{*}{$\begin{array}{l}\text { All Patients } \\
\text { Total } \\
(\mathrm{N}=140,903)\end{array}$} & \multicolumn{3}{|c|}{ Patients With Type 2 Diabetes ${ }^{b}$} & \multicolumn{4}{|c|}{ Patients Without Diabetes } \\
\hline & & $\begin{array}{c}\text { Total } \\
(\mathrm{n}=38,553)\end{array}$ & $\begin{array}{c}\text { With } \\
\text { Recurrence } \\
(\mathrm{n}=10,096)\end{array}$ & $\begin{array}{l}\text { Without } \\
\text { Recurrence } \\
(\mathrm{n}=28,457)\end{array}$ & $\begin{array}{c}\text { Total } \\
(\mathrm{n}=81,845)\end{array}$ & $\begin{array}{c}\text { With } \\
\text { Recurrence }^{c} \\
(\mathrm{n}=15,574)\end{array}$ & $\begin{array}{l}\text { Without } \\
\text { Recurrence } \\
(\mathrm{n}=66,271)\end{array}$ & $P$ Value ${ }^{\mathrm{d}}$ \\
\hline Age, years, mean (SD) & $66.8(14.6)$ & $68.2(12.9)$ & $72.2(12.2)$ & $66.8(12.8)$ & $65.6(15.4)$ & $72.5(15.1)$ & $64.0(15.1)$ & $<0.001$ \\
\hline \multicolumn{9}{|l|}{ Gender, n (\%) } \\
\hline Male & $82,517(58.6)$ & $22,689(58.9)$ & $5,642(55.9)$ & $17,047(59.9)$ & $48,026(58.7)$ & $8,595(55.2)$ & $39,431 \quad(59.5)$ & 0.571 \\
\hline \multicolumn{9}{|l|}{ Mortality, n (\%) } \\
\hline All-cause & $12,798 \quad(9.1)$ & $4,238(11.0)$ & $1,289(12.8)$ & $2,949(10.4)$ & 6,502 & $1,575(10.1)$ & $4,927 \quad(7.4)$ & \\
\hline CHD-related & $8,482 \quad(6.0)$ & $2,929 \quad(7.6)$ & $2,929(29.0)$ & - & $4,435 \quad(5.4)$ & $4,435(28.5)$ & - & \\
\hline \multicolumn{9}{|c|}{ Major baseline comorbidities, n (\%) } \\
\hline Hypertension & $85,164(60.4)$ & $28,809(74.7)$ & $7,851 \quad(77.8)$ & $20,958(73.7)$ & $42,949(52.5)$ & $9,499(61.0)$ & $33,450(50.5)$ & $<0.001$ \\
\hline Dyslipi & $65,283(46.3)$ & $22,172(57.5)$ & $5,597(55.4)$ & $16,575(58.3)$ & $33,295(40.7)$ & $6,138 \quad(39.4)$ & $27,157 \quad(41.0)$ & $<0.001$ \\
\hline Coronary artery & $49,212(34.9)$ & $17,891 \quad(46.4)$ & $5,330(52.8)$ & $12,561 \quad(44.1)$ & $23,481 \quad(28.7)$ & $5,623(36.1)$ & $17,858 \quad(27.0)$ & $<0.001$ \\
\hline Dyspnea & $35,734 \quad(25.4)$ & $12,166(31.6)$ & $3,701 \quad(36.7)$ & $8,465(29.8)$ & $17,729(21.7)$ & $4,090(26.3)$ & $13,639(20.6)$ & $<0.001$ \\
\hline Heart failure & $22,428(15.9)$ & $9,361 \quad(24.3)$ & $3,426(33.9)$ & $5,935(20.9)$ & $9,387 \quad(11.5)$ & $2,926(18.8)$ & $6,461 \quad(9.8)$ & $<0.001$ \\
\hline Anemia & $22,485(16.0)$ & $8,793(22.8)$ & $2,988 \quad(29.6)$ & $5,805 \quad(20.4)$ & $10,012(12.2)$ & $2,825(18.1)$ & $7,187 \quad(10.8)$ & $<0.001$ \\
\hline Rena & $15,032(10.7)$ & $7,456(19.3)$ & $2,699(26.7)$ & $4,757(16.7)$ & $5,321 \quad(6.5)$ & 1,707 (11.0) & $3,614 \quad(5.5)$ & 1 \\
\hline $\begin{array}{l}\text { Length of stay, index } \\
\text { event, mean days (SD) }\end{array}$ & $6.2(11.8)$ & $7.6(13.7)$ & $8.9(14.4)$ & $7.2(13.4)$ & $5.2 \quad(9.3)$ & $6.3 \quad(9.3)$ & $4.9 \quad(9.3)$ & $<0.001$ \\
\hline \multicolumn{9}{|l|}{ Admitting diagnosis, $\mathbf{n}(\%)$} \\
\hline Unst & $60,152 \quad(42.7)$ & $16,753(43.5)$ & $2,791 \quad(27.6)$ & $13,962 \quad(49.1)$ & $34,530(42.2)$ & $3,495(22.4)$ & $31,035(46.8)$ & $<0.001$ \\
\hline STEMI & $58,103 \quad(41.2)$ & $15,124(39.2)$ & $5,116(50.7)$ & $10,008(35.2)$ & $34,578(42.3)$ & $9,082(58.3)$ & $25,496(38.5)$ & $<0.001$ \\
\hline NSTEMI & $22,648(16.1)$ & $6,676 \quad(17.3)$ & $2,189(21.7)$ & $4,487(15.8)$ & $12,737(15.6)$ & $2,997(19.2)$ & $9,740 \quad(14.7)$ & $<0.001$ \\
\hline \multicolumn{9}{|c|}{ Services during index hospitalization, $\mathbf{n}(\%)$} \\
\hline PCI only & $43,329(30.8)$ & $10,970(28.5)$ & $2,731 \quad(27.1)$ & $8,239(29.0)$ & $26,200(32.0)$ & $5,110(32.8)$ & $21,090(31.8)$ & $<0.001$ \\
\hline CABG only & $12,478 \quad(8.9)$ & $4,203(10.9)$ & $737 \quad(7.3)$ & $3,466(12.2)$ & $6,321 \quad(7.7)$ & $845 \quad(5.4)$ & $5,476 \quad(8.3)$ & $<0.001$ \\
\hline $\mathrm{PCI}$ and $\mathrm{CABG}$ & $1,003 \quad(0.7)$ & $274 \quad(0.7)$ & $63 \quad(0.6)$ & $211 \quad(0.7)$ & $570 \quad(0.7)$ & $128 \quad(0.8)$ & $442 \quad(0.7)$ & $<0.001$ \\
\hline \multicolumn{9}{|c|}{$\begin{array}{l}\text { ¿Recurrence is defined as occurrence of nonfatal myocardial infarction, nonfatal stroke, or CHD-related mortality after the index ACS event. } \\
\text { dStatistical comparison between patients with type } 2 \text { diabetes and patients without type } 2 \text { diabetes. } \\
\text { ACS = acute coronary syndrome; } C A B G=\text { coronary artery bypass graft; } C H D=\text { coronary heart disease; NSTEMI=non-ST segment elevation myocardial infarction; } \\
P C I=\text { percutaneous coronary intervention; SD = standard deviation; STEMI =ST segment elevation myocardial infarction. }\end{array}$} \\
\hline
\end{tabular}

discontinuation was defined as a gap in treatment of 45 days or longer in each medication based on days of supply. Rates of adherence to medications were compared across medications and by the presence or absence of recurrent CVEs.

Medication classes included guideline-recommended cardiovascular treatments (angiotensin-converting enzyme inhibitors/angiotensin receptor blockers, beta-blockers, antiplatelet agents, and statins) and other commonly used therapies such as antihypertensives, anticoagulants, antidyslipidemia, and antidiabetic medications (oral and parenteral). Treatment to clinical standard was defined as postdischarge fill within 30 days of an antiplatelet, anticoagulant, anti-ischemic (beta-blocker or calcium channel blocker), antihypertensive, or antidyslipidemia medication. For patients with type 2 diabetes, minimum treatment included medications targeted at glycemic control.
Persistence was measured as the length of treatment from the start of therapy to either the end of therapy (according to the minimum time recommended in the American College of Cardiology/American Heart Association [ACC/AHA] guidelines $)^{10}$ or to a gap of 45 days, whichever occurred sooner. The total length of therapy was described as the lengths of followup (i.e., patients with at least 1 year and 3 years of follow-up). Treatment discontinuation was defined as a gap in treatment of 45 days or longer in each medication class based on days of supply. Rates of adherence and persistence with each medication were compared across medications and by the presence or absence of subsequent CVEs.

\section{Statistical Analysis}

Descriptive statistics included means (standard deviations) and relative frequencies for continuous and categorical data, 


\begin{tabular}{|c|c|c|c|c|c|c|}
\hline & \multicolumn{3}{|c|}{ Patients With Type 2 Diabetes $^{a}$} & \multicolumn{3}{|c|}{ Patients Without Diabetes } \\
\hline & Total & $\begin{array}{l}\text { Treatment } \\
\text { to Clinical } \\
\text { Standard }{ }^{\mathrm{b}}\end{array}$ & $\begin{array}{c}\text { Treatment Not } \\
\text { to Clinical } \\
\text { Standard }\end{array}$ & Total & $\begin{array}{l}\text { Treatment } \\
\text { to Clinical } \\
\text { Standard }{ }^{\mathrm{b}}\end{array}$ & $\begin{array}{l}\text { Treatment Not } \\
\text { to Clinical } \\
\text { Standard }\end{array}$ \\
\hline Patients with recurrence during follow up, n (\%) & $10,096(26.2)$ & $4,660(22.4)$ & $5,436(30.7)$ & $15,574(19.0)$ & $8,893(16.3)$ & $6,681(34.6)$ \\
\hline Time to first recurrence, years, mean (SD) & $0.8 \quad(1.1)$ & $1.0 \quad(1.1)$ & $0.7 \quad(1.0)$ & $0.7 \quad(1.1)$ & $0.9 \quad(1.1)$ & $0.5 \quad(1.0)$ \\
\hline \multicolumn{7}{|l|}{ First recurrent event type, n (\%) } \\
\hline Myocardial infarction & $6,589(65.3)$ & $3,627(77.8)$ & $2,962(54.5)$ & $10,354(66.5)$ & $7,368(82.9)$ & $2,986(44.7)$ \\
\hline CHD-rel & $2,029(20.1)$ & $277 \quad(5.9)$ & $1,752(32.2)$ & $3,562(22.9)$ & $492 \quad(5.5)$ & $3,070(46.0)$ \\
\hline Nonfatal stroke & $1,478(14.6)$ & $756(16.2)$ & $722(13.2)$ & $1,658(10.7)$ & $1,033(11.6)$ & $625 \quad(9.4)$ \\
\hline \multicolumn{7}{|l|}{ Intervention at first recurrence, $\mathrm{n}(\%)$} \\
\hline No intervention & $8,000(79.2)$ & $3,349(71.9)$ & $4,651(85.6)$ & $12,067(77.5)$ & $6,063(68.2)$ & $6,004(89.9)$ \\
\hline Coronary stent & $1,972(19.5)$ & $1,272(27.3)$ & $700(12.9)$ & $3,601(23.1)$ & $2,963(33.3)$ & $638 \quad(9.5)$ \\
\hline Catheter & $1,639(16.2)$ & $1,016(21.8)$ & $623(11.5)$ & $2,781 \quad(17.9)$ & $2,239(25.2)$ & $542 \quad(8.1)$ \\
\hline Primary a & $888 \quad(8.8)$ & $602(12.9)$ & $286 \quad(5.3)$ & $1,689(10.8)$ & $1,447(16.3)$ & $242 \quad(3.6)$ \\
\hline $\mathrm{CABG}$ & $380 \quad(3.8)$ & $238 \quad(5.1)$ & $142 \quad(2.6)$ & $507 \quad(3.3)$ & $411 \quad(4.6)$ & $96 \quad(1.4)$ \\
\hline Patients with & 22,588 & 13,640 & 8,948 & 48,358 & 34,871 & 13,487 \\
\hline Patients with subsequent event during follow-up, n (\%) & $6,106(27.0)$ & $3,462(25.4)$ & $2,644(29.6)$ & $9,069(18.8)$ & $6,520(18.7)$ & $2,549(18.9)$ \\
\hline \multicolumn{7}{|l|}{ Cardiovascular rehospitalizations, $\mathbf{n}$, mean (SD) } \\
\hline Within 30 days & $0.3 \quad(0.6)$ & $0.3 \quad(0.6)$ & $0.3 \quad(0.6)$ & $0.3 \quad(0.6)$ & $0.3 \quad(0.6)$ & $0.3 \quad(0.6)$ \\
\hline Within 1 & $1 \quad(1.2)$ & $1.0 \quad(1.2)$ & $1.0 \quad(1.3)$ & $0.8 \quad(1.0)$ & $0.8 \quad(1.0)$ & $0.8 \quad(1.1)$ \\
\hline Within 365 days & $1.7 \quad(1.8)$ & $1.6 \quad(1.7)$ & $1.8 \quad(1.9)$ & $1.3 \quad(1.3)$ & $1.2(1.3)$ & $1.3 \quad(1.4)$ \\
\hline Time to first recurrence, years, mean (SD) & $1.2(1.2)$ & $1.2(1.2)$ & $1.2(1.2)$ & $1.2(1.2)$ & $1.1 \quad(1.2)$ & $1.3 \quad(1.2)$ \\
\hline Patients with at least 36 months of eligibility, $\mathrm{n}$ & 9,160 & 5,683 & 3,477 & 19,753 & 14,316 & 5,437 \\
\hline Patients with subsequent event during follow-up, n (\%) & $2,706(29.5)$ & $1,615(28.4)$ & $1,091 \quad(31.4)$ & $4,264(21.6)$ & $3,106(21.7)$ & $1,158(21.3)$ \\
\hline \multicolumn{7}{|l|}{ Cardiovascular rehospitalizations, $\mathbf{n}$, mean (SD) } \\
\hline Within 1 year & $1.3(1.6)$ & $1.3 \quad(1.5)$ & $1.3(1.7)$ & $1.0 \quad(1.1)$ & $1.0 \quad(1.1)$ & $1.0 \quad(1.1)$ \\
\hline Within 2 years & $2.3 \quad(2.4)$ & $2.2(2.3)$ & $2.4 \quad(2.5)$ & $1.6 \quad(1.6)$ & $1.6 \quad(1.6)$ & $1.7 \quad(1.7)$ \\
\hline Within 3 years & $3.4 \quad(3.7)$ & $3.3(3.5)$ & $3.6 \quad(4.0)$ & $2.4 \quad(2.3)$ & $2.3 \quad(2.3)$ & $2.7 \quad(2.4)$ \\
\hline Time to first recurrence, years, mean (SD) & $1.8 \quad(1.4)$ & $1.7 \quad(1.4)$ & $1.8 \quad(1.4)$ & $1.6 \quad(1.4)$ & $1.6 \quad(1.4)$ & $1.8 \quad(1.4)$ \\
\hline \multicolumn{7}{|c|}{$\begin{array}{l}\text { aPatients with type } 2 \text { diabetes were defined as having } \geq 2 \text { claims of type } 2 \text { diabetes } \geq 30 \text { days apart; } \geq 1 \text { claim of type } 2 \text { diabetes and } \geq 1 \text { claim for an antidiabetic medica- } \\
\text { tion; or } \geq 2 \text { prescriptions of antidiabetic medications. } \\
\text { bTreatment to clinical standard is defined as postdischarge prescription fill within } 30 \text { days of an antiplatelet, anticoagulant, anti-ischemic (beta blocker or calcium channel } \\
\text { blocker), antihypertensive, or antidyslipidemia medication. For patients with diabetes, minimum treatment also includes medications required for glycemic control. } \\
{ }^{c} C H D \text {-related mortality is defined as presence of a diagnosis code for coronary heart disease } 7 \text { days prior to date of death. } \\
C A B G=\text { coronary artery bypass graft; } C H D=\text { coronary heart disease; } S D=\text { standard deviation. }\end{array}$} \\
\hline
\end{tabular}

respectively. Descriptive analysis was performed for all variables in the study. Univariate statistics were calculated, and bivariate analyses were used to explore the relationships between ACS and medication use and clinically significant covariates. ${ }^{11-13}$ Subgroup comparisons were performed using t-tests for continuous data and $\chi^{2}$ tests for categorical data. The association between preventive medications and subsequent CVEs were assessed using $\chi^{2}$ tests and multiple comparisons ANOVA adjusted for relevant cofactors. A Cox proportional hazards regression model was used to predict the time to recurrence.

\section{Results}

\section{Baseline Patient Characteristics}

A total of 140,903 ACS patients were included in the study (Table 1). Of these, 38,553 (27.4\%) had type 2 diabetes. The mean age of the total population was 66.8 years (68.2 years type 2 diabetes cohort, 65.6 years nondiabetes cohort). The mean length of follow-up was 1.9 years overall (1.8 years in the type 2 diabetes and nondiabetes cohorts).

At baseline, the most common comorbidities in the overall population were hypertension (60.4\%), dyslipidemia (46.3\%), coronary artery disease (34.9\%), dyspnea (25.4\%), and heart failure (15.9\%). These baseline comorbidities were statistically significantly higher among patients in the type 2 diabetes group compared with the nondiabetes group (hypertension $74.7 \%$ vs. $52.5 \%$; dyslipidemia $57.5 \%$ vs. $40.7 \%$; coronary artery disease $46.4 \%$ vs. $28.7 \%$; and heart failure $24.3 \%$ vs. $11.5 \%$, respectively; $P<0.001$ for all comparisons; Table 1).

\section{Index ACS Characteristics}

During the index hospitalization for ACS, $42.7 \%$ of patients overall had UA, and $40.4 \%$ had coronary artery bypass graft 
Clinical Outcomes and Medication Adherence in Acute Coronary Syndrome Patients

With and Without Type 2 Diabetes Mellitus: A Longitudinal Analysis 2006-2011

TABLE 3 Medication Adherence at 1 and 3 Years of Follow-up

\begin{tabular}{|c|c|c|c|c|c|c|c|c|c|}
\hline & \multirow{2}{*}{\multicolumn{2}{|c|}{$\begin{array}{l}\text { All Patients } \\
\\
\text { Totalb }^{\mathrm{b}} \\
\text { n (\%) }\end{array}$}} & \multicolumn{3}{|c|}{ Patients With Type 2 Diabetes $^{a}$} & \multicolumn{3}{|c|}{ Patients Without Diabetes } & \multirow[b]{2}{*}{$\begin{array}{c}P \\
\text { Value }^{\mathrm{i}}\end{array}$} \\
\hline & & & $\begin{array}{l}\text { Totalc } \\
\text { n (\%) }\end{array}$ & $\begin{array}{c}\text { With } \\
\text { Recurrence } \\
\text { n (\%) }\end{array}$ & $\begin{array}{c}\text { Without } \\
\text { Recurrence } \\
\text { n (\%) }\end{array}$ & $\begin{array}{l}\text { Total } \\
\text { n (\%) }\end{array}$ & $\begin{array}{c}\text { With } \\
\text { Recurrenceg } \\
\text { n (\%) }\end{array}$ & $\begin{array}{c}\text { Without } \\
\text { Recurrence } \\
\text { n (\%) }\end{array}$ & \\
\hline \multicolumn{10}{|c|}{ Antihypertensives } \\
\hline 1 year & 56,575 & $(66.5)$ & $16,059 \quad(71.1)$ & $4,200 \quad(68.8)$ & $11,859 \quad(72.0)$ & $31,260 \quad(64.6)$ & $6,055 \quad(66.8)$ & $25,205 \quad(64.2)$ & $<0.001$ \\
\hline 3 years & 23,808 & $(67.0)$ & $6,614 \quad(72.2)$ & $1,882 \quad(69.6)$ & $4,732 \quad(73.3)$ & $12,807 \quad(64.8)$ & $2,857 \quad(67.0)$ & $9,950 \quad(64.2)$ & $<0.001$ \\
\hline \multicolumn{10}{|l|}{ ACE/ARB } \\
\hline 1 year & 26,479 & $(31.1)$ & $7,874 \quad(34.9)$ & $2,050 \quad(33.6)$ & $5,824 \quad(35.4)$ & $14,442 \quad(29.9)$ & $3,181 \quad(35.1)$ & $11,261 \quad(28.7)$ & $<0.001$ \\
\hline 3 years & 11,229 & $(13.2)$ & $3,305 \quad(36.1)$ & $935 \quad(34.6)$ & $2,370 \quad(36.7)$ & $5,940 \quad(30.1)$ & $1,498 \quad(35.1)$ & $4,442 \quad(28.7)$ & $<0.001$ \\
\hline \multicolumn{10}{|c|}{ Beta-blocker } \\
\hline 1 year & 45.779 & $(53.8)$ & $12,746 \quad(56.4)$ & $3,442 \quad(56.4)$ & $9,304 \quad(56.5)$ & $25,589 \quad(52.9)$ & $5,142 \quad(56.7)$ & $20,447 \quad(52.0)$ & $<0.001$ \\
\hline 3 years & 19,339 & $(54.4)$ & $5,217 \quad(57.0)$ & $1,550 \quad(57.3)$ & $3,667 \quad(56.8)$ & $10,564 \quad(53.5)$ & $2,455 \quad(57.6)$ & $8,109 \quad(52.3)$ & $<0.001$ \\
\hline \multicolumn{10}{|l|}{ Statins } \\
\hline 1 year & 42,787 & $(50.3)$ & $11,735 \quad(52.0)$ & $2,931 \quad(48.0)$ & $8,804 \quad(53.4)$ & $24,289 \quad(50.2)$ & $4,620 \quad(50.9)$ & $19,669 \quad(50.1)$ & 0.039 \\
\hline 3 years & 17,705 & $(49.8)$ & $4,712 \quad(51.4)$ & $1,300 \quad(48.0)$ & $3,412 \quad(52.9)$ & $9,863 \quad(49.9)$ & $2,162 \quad(50.7)$ & $7,701 \quad(49.7)$ & 0.876 \\
\hline \multicolumn{10}{|c|}{ Antiplatelets } \\
\hline 1 year & 33,973 & $(39.9)$ & $9,263 \quad(41.0)$ & $2,634 \quad(43.1)$ & $6,629 \quad(40.2)$ & $19,332 \quad(40.0)$ & $4,242 \quad(46.8)$ & $15,090 \quad(38.4)$ & $<0.001$ \\
\hline 3 years & 14,572 & $(41.0)$ & $3,891 \quad(42.5)$ & $1,227 \quad(45.3)$ & $2,664 \quad(41.3)$ & $8,039 \quad(40.7)$ & $2,054 \quad(48.2)$ & $5,985 \quad(38.6)$ & 0.298 \\
\hline \multicolumn{10}{|c|}{ Oral antidiabetics } \\
\hline 1 year & 11,959 & $(14.1)$ & $11,452 \quad(50.7)$ & $2,640 \quad(43.2)$ & $8,812 \quad(53.5)$ & - & - & - & $<0.001$ \\
\hline 3 years & 5,097 & $(14.3)$ & $4,873 \quad(53.2)$ & $1,273 \quad(47.0)$ & $3,600 \quad(55.8)$ & - & - & - & $<0.001$ \\
\hline \multicolumn{10}{|c|}{ slipidemicsj } \\
\hline 1 year & 11,379 & (13.4) & $4,011 \quad(17.8)$ & $973 \quad(15.9)$ & $3,038 \quad(18.4)$ & $5,428 \quad(11.2)$ & $987 \quad(10.9)$ & $4,441 \quad(11.3)$ & 0.186 \\
\hline 3 years & 5,325 & $(15.0)$ & $1,803 \quad(19.7)$ & $490 \quad(18.1)$ & $1,313 \quad(20.3)$ & $2,536 \quad(12.8)$ & $548 \quad(12.9)$ & $1,988 \quad(12.8)$ & 0.122 \\
\hline \multicolumn{10}{|c|}{ Other antihypertensives } \\
\hline 1 year & 26,368 & (31.0) & $9,380 \quad(41.5)$ & $2,754 \quad(45.1)$ & $6,626 \quad(40.2)$ & $12,413 \quad(25.7)$ & $2,691 \quad(29.7)$ & $9,722 \quad(24.7)$ & $<0.001$ \\
\hline 3 years & 11,007 & $(31.0)$ & $3,826 \quad 41.8)$ & $1,230 \quad(45.6)$ & $2,596 \quad(40.2)$ & $5,061 \quad(25.6)$ & $1,220 \quad(28.6)$ & $3,841 \quad(24.8)$ & $<0.001$ \\
\hline \multicolumn{10}{|c|}{ Anticoagulants } \\
\hline 1 year & 7,453 & $(8.8)$ & $2,259 \quad(10.0)$ & $695 \quad(11.4)$ & 1,564 & 3,704 & $957 \quad(10.6)$ & 2,747 & 0.010 \\
\hline 3 years & 3,089 & $(8.7)$ & $(9.7)$ & $286 \quad(10.6)$ & $(9.3)$ & 1,507 & $(9.1)$ & 1,118 & 0.170 \\
\hline \multicolumn{10}{|l|}{ Insulin } \\
\hline 1 year & 5,297 & $(6.2)$ & $5,111 \quad(22.6)$ & $1,567 \quad(25.7)$ & $3,544 \quad(21.5)$ & - & - & - & $<0.001$ \\
\hline 3 years & 2,008 & $(5.7)$ & $1,929 \quad(21.1)$ & $665 \quad(24.6)$ & $1,264 \quad(19.6)$ & - & - & - & $<0.001$ \\
\hline \multicolumn{10}{|c|}{ 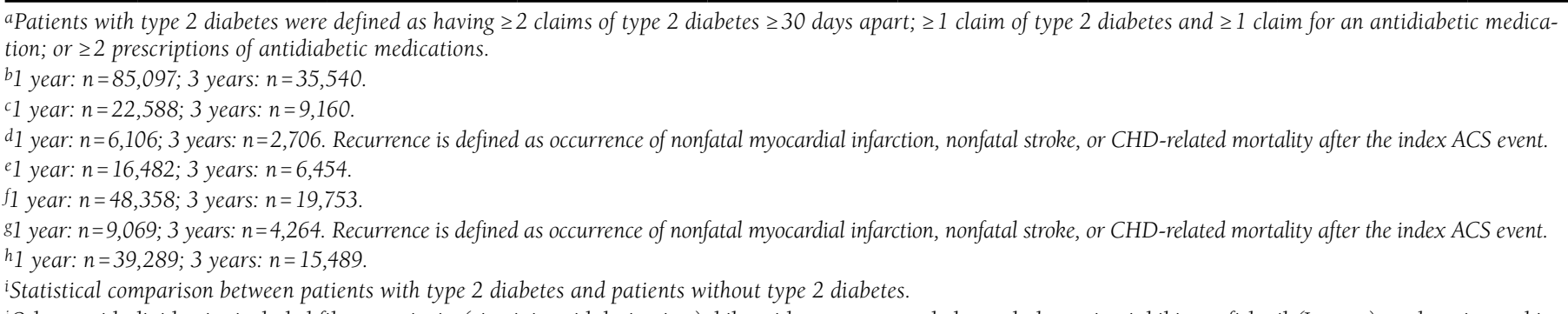 } \\
\hline
\end{tabular}

(CABG) and/or percutaneous coronary intervention (PCI). A larger proportion of patients in the type 2 diabetes group had UA compared with the nondiabetes group $(43.5 \%$ vs. $42.2 \%$, $P<0.001)$. A slightly smaller but statistically significant proportion of patients in the type 2 diabetes group had CABG and/or PCI compared with the nondiabetes group (40.1\% vs. $40.4 \%$, $P<0.001)$ at the index hospitalization (Table 1).

\section{Subsequent Cardiovascular Events}

During the follow-up period, 10,096 patients (26.2\%) in the type 2 diabetes group had a subsequent CVE, compared with 15,574 patients (19.0\%) in the nondiabetes group (Table 2). The mean time to first CVE was 0.8 years for the type 2 diabetes group and 0.7 years for the nondiabetes group. In both cohorts, MI was the first subsequent CVE for the majority of 
Clinical Outcomes and Medication Adherence in Acute Coronary Syndrome Patients

With and Without Type 2 Diabetes Mellitus: A Longitudinal Analysis 2006-2011

TABLE 4 Treatment Persistence Days at 1 and 3 Years of Follow-upa

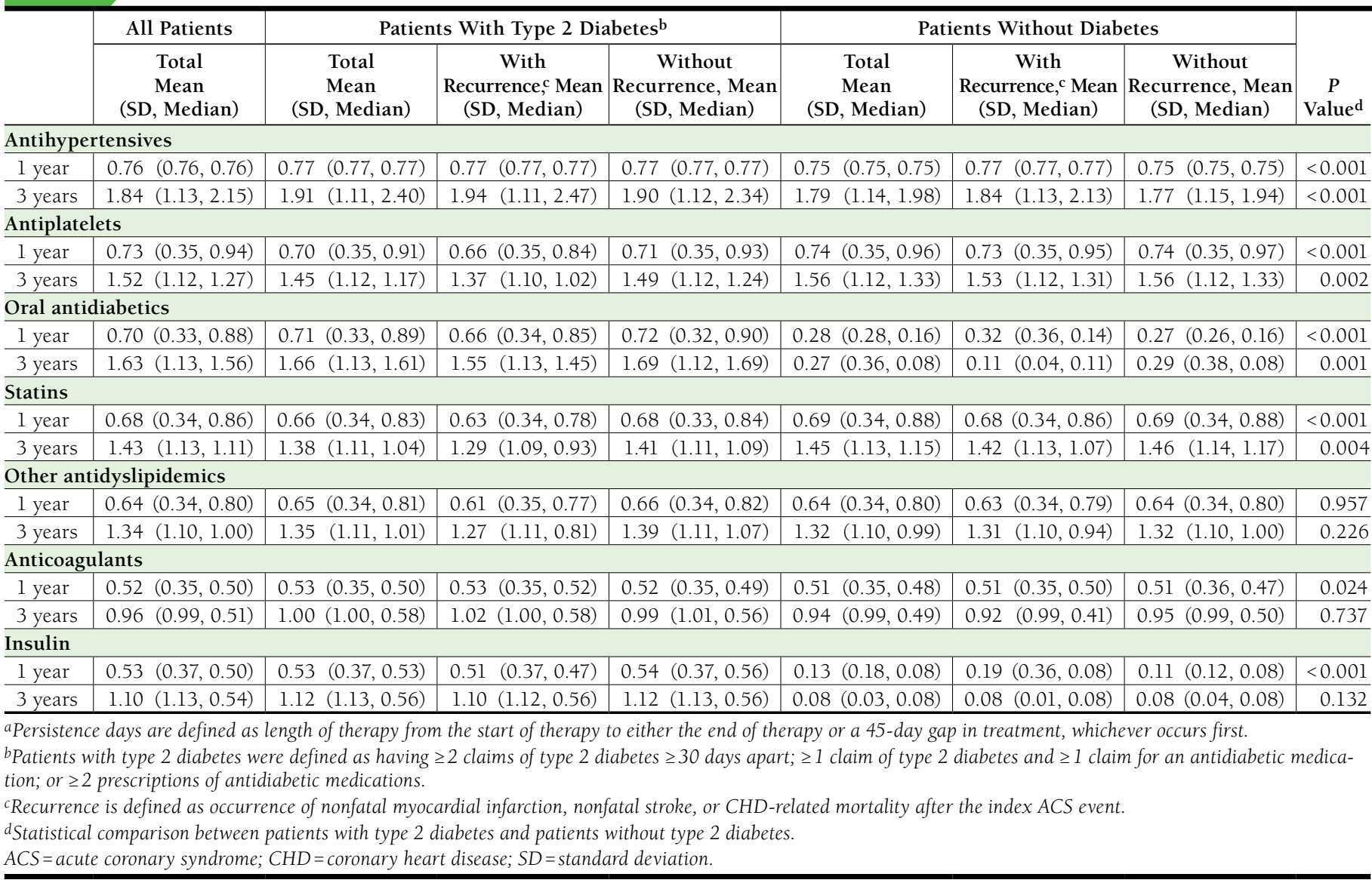

patients (65.3\% type 2 diabetes group vs. $66.5 \%$ nondiabetes group). The majority of patients in both cohorts received no revascularization at the first recurrence following index ACS discharge (79.2\% type 2 diabetes group vs. $77.5 \%$ nondiabetes group). Among those who underwent revascularization at first recurrence following index ACS discharge, coronary stenting was the most common procedure (19.5\% in the type 2 diabetes group vs. 23.1 in the nondiabetes group).

\section{Cardiovascular Event Recurrence}

Patients with diabetes who received treatment to clinical standard had a lower rate of CVE recurrence compared with patients who did not receive treatment to clinical standard (Table 2). This was consistent for patients in the nondiabetes cohort overall and for 1 year of follow-up. Mean time to recurrence did not differ between the type 2 diabetes and nondiabetes cohorts or between patients who received treatment to clinical standard and those who did not receive treatment to clinical standard.

\section{Adherence and Persistence}

Adherence rates, as measured by MPR, were not statistically different between patients with and without type 2 diabetes for anticoagulants, statins, or other antidyslipidemics at 1 or 3 years of follow-up or for antiplatelet agents at 3 years of follow-up. However, patients with type 2 diabetes had statistically higher adherence rates for antiplatelet agents at 1 year of follow-up and for antihypertensives at 1 and 3 years of followup, compared with patients without diabetes (Table 3).

Patients with type 2 diabetes were more persistent with antihypertensive therapy, as measured by duration of therapy, compared with patients without diabetes (Table 4). At 1 and 3 years of follow-up, for antihypertensive medications, patients with type 2 diabetes had significantly more days of persistence than those without diabetes. However, patients without diabetes had greater persistence for antiplatelet agents and statins at 1 and 3 years of follow-up. Persistence rates for antidyslipidemics and anticoagulants were not significantly different between the 2 groups at 3 years of follow-up, although persistence with anticoagulants was significantly different at 1 year of follow-up. 


\section{Discussion}

In this real-world analysis of patients who had been hospitalized with an ACS event, patients with type 2 diabetes had a higher rate of subsequent CVEs than those without diabetes. These findings are consistent with previous research in smaller cohorts and are plausible given the increased prevalence of other cardiovascular risk factors in patients with type 2 diabetes. ${ }^{14-16}$

The majority of patients in the type 2 diabetes and nondiabetes cohorts did not undergo revascularization (PCI or $\mathrm{CABG}$ ) at the time of the initial ACS event. Intensive secondary preventive measures are recommended for patients with type 2 diabetes, so our results may suggest underutilization of revascularization for these patients. ${ }^{17}$ However, it is worth noting that a sizeable proportion of the study population (42.7\%) had UA, for whom medication and noninvasive testing are recommended before catheterization or revascularization procedures unless the patients are at high risk for clinical events. ${ }^{18}$ Following ACS hospitalization, the patients who received standard clinical therapy according to ACC/AHA ${ }^{10}$ and American Diabetes Association guidelines ${ }^{19}$ (for patients with type 2 diabetes) had lower rates of CVE recurrence and a longer time to recurrence compared with those who did not receive treatment, which is consistent with previous studies. ${ }^{16,20}$

In general, adherence rates were not statistically different between patients with and without type 2 diabetes, but persistence was higher among patients with type 2 diabetes. Our results indicate that adherence and persistence rates show room for improvement. The lack of a difference in adherence rates between the type 2 diabetes and nondiabetes cohorts differs from previous studies that reported type 2 diabetes was associated with lower adherence rates in patients with ACS..$^{21,22}$ A possible explanation for the difference in adherence rates between the current and prior studies is that in the current study, patients were stratified based on their type 2 diabetes status. In contrast, earlier studies stratified patients according to ACS treatment and assessed the effect of type 2 diabetes as a subanalysis. ${ }^{21,22}$ Furthermore, the current study is unique in that a large patient population was followed for an extended period, unlike prior studies, which used smaller populations with follow-up periods of 12 months or less. ${ }^{21,22}$ Interestingly, insulin adherence was higher among patients in the type 2 diabetes cohort who had recurrent ACS events compared with those without recurrence. Insulin use may be a marker of a longer duration of disease-that is, patients taking insulin typically have more advanced or severe disease than those who are not receiving insulin therapy who are able to manage their condition with oral agents alone.

Although guidelines such as those for the management of ACS are based on clinical trial data, the applicability of guidelines may be reduced substantially by factors not present in a clinical trial situation, for example, nonadherence. In contrast, the findings of the current study are based on real-world experience and reflect how precisely patients follow treatment regimens outside of a tightly controlled research environment. Treatment adherence is an important consideration when evaluating outcomes. For example, patients with type 2 diabetes remain at increased risk for subsequent CVEs following ACS even when they receive intensive standard-of-care treatment. ${ }^{9}$ The question is whether the increased risk is due to an inadequate standard of care or to poor treatment adherence. Treatment recommendations could be strengthened by focusing not only on what medications to prescribe but also on how to address noncompliance and improve adherence.

\section{Limitations}

The limitations of this study must be considered in interpreting its results. The data for the study were obtained from medical and pharmacy claims, which lack information pertaining to severity of type 2 diabetes and the degree to which type 2 diabetes was successfully managed. Furthermore, although the presence or absence of some other cardiovascular risk factors can be determined from claims analysis, it is not possible to measure the degree of risk or the success of management of these conditions (e.g., hypertension and hypercholesterolemia). Some other important cardiovascular risk factors, such as tobacco use, cannot be adequately addressed from claims analysis. Information on patients' weight and body mass index are also absent from claims. Patients with type 2 diabetes were defined as those who had 2 or more claims of type 2 diabetes at least 30 days apart; 1 or more claims of type 2 diabetes plus 1 or more claims for an antidiabetic medication; or 2 or more prescriptions for antidiabetic medications. This definition did not capture patients who had ICD-9-CM codes for type 1 diabetes or those with ICD-9-CM codes for type 2 diabetes but who did not satisfy other aspects of the definition of type 2 diabetes used for this study. Thus, some patients with type 2 diabetes may have inadvertently been placed into the nondiabetes cohort. Additionally, administrative claims may have contained undetected coding errors or omissions. All patients included in this study were members of large commercial health plans in the United States, and as such, findings may not be generalizable to patients with other types of insurance or to those living outside of the United States. The data regarding medication adherence and persistence may be inaccurate for patients who were treated with sample medications or through other programs that do not generate pharmacy claims. Furthermore, adherence and persistence with nonprescription medications (most notably aspirin) could not be addressed. Lastly, medications may have been prescribed for different durations for patients with and without diabetes. Differences in persistence and adherence rates may be a reflection of these different dosing regimens rather than an indication of adherence. 


\section{Clinical Outcomes and Medication Adherence in Acute Coronary Syndrome Patients With and Without Type 2 Diabetes Mellitus: A Longitudinal Analysis 2006-2011}

\section{Conclusions}

This study provides contemporary, long-term data on the impact of type 2 diabetes on cardiovascular risk in a large population. Although patients with type 2 diabetes had a higher risk of subsequent CVEs following an initial ACS event compared with patients without diabetes, the rate of CVE recurrence was lower for patients who received standard-ofcare treatment. Despite observed treatment persistence among patients with diabetes, medication adherence rates remained suboptimal in this group, indicating a continuing need for patient education. Focusing attention on guideline and medication adherence, particularly for patients at high risk, may be as important a strategy for reducing the burden of care as advancing the science that informs the practice guidelines.

\section{Authors}

MARK J. CZIRAKY, PharmD, CLS, is Vice President of Research Health Outcomes, and RAKESH LUTHRA, MS, was Research Analyst, at the time of this study, HealthCore, Wilmington, Delaware. MAXINE D. FISHER, PhD, is Director, Real World Evidence, Vector Oncology, Memphis, Tennessee; VANESSA S. REDDY, MS, is with F. Hoffmann-La Roche, Basel, Switzerland; and THOMAS P. POWER, $M D$, is Medical Director, AIM Specialty Health, Deerfield, Illinois. YAPING XU, MD, MPH, and KENNETH WILHELM, MD, are employees of Genentech, South San Francisco, California.

AUTHOR CORRESPONDENCE: Mark J. Cziraky, PharmD, CLS, 800 Delaware Ave., 5th Fl., Wilmington, Delaware 19801.

Tel.: 302.230.2110; Fax: 302.230.2020;

E-mail:mcziraky@healthcore.com.
3. Stone GW, Maehara A, Lansky AJ, et al. A prospective natural-history study of coronary atherosclerosis. N Engl J Med. 2011;364(3):226-35.

4. Donnan PT, Boyle DIR, Broomhall J, et al. Prognosis following first acute myocardial infarction in type 2 diabetes: a comparative population study. Diabet Med. 2002;19(6):448-55.

5. Haffner SM, Lehto S, Ronnemaa T, Pyorala K, Laakso M. Mortality from coronary heart disease in subjects with type 2 diabetes and in nondiabetic subjects with and without prior myocardial infarction. N Engl J Med. 1998;339(4):229-34.

6. Grundy SM, Benjamin IJ, Burke GL, et al. Diabetes and cardiovascular disease: a statement for healthcare professionals from the American Heart Association. Circulation. 1999;100(10):1134-46

7. Taylor KS, Heneghan CJ, Farmer AJ, et al. All-cause and cardiovascular mortality in middle-aged people with type 2 diabetes compared with people without diabetes in a large U.K. primary care database. Diabetes Care. 2013;36(8):2366-71.

8. Bartnik M, Malmberg K, Ryden L. Management of patients with type 2 diabetes after acute coronary syndromes. Diab Vasc Dis Res. 2005;2(3):144-54. 9. Ahmed S, Cannon CP, Murphy SA, Braunwald E. Acute coronary syndromes and diabetes: Is intensive lipid lowering beneficial? Results of the PROVE IT-TIMI 22 trial. Eur Heart J. 2006;27(19):2323-29.

10. O'Gara PT, Kushner FG, Ascheim DD, et al. 2013 ACCF/AHA guideline for the management of ST-elevation myocardial infarction: a report of the American College of Cardiology Foundation/American Heart Association Task Force on Practice Guidelines. Circulation. 2013;127(4):e362-425.

11. Zhao Z, Zhu B, Anderson J, Fu H, LeNarz L. Resource utilization and healthcare costs for acute coronary syndrome patients with and without diabetes mellitus. J Med Econ. 2020;13(4):748-59.

12. Amed S, Vanderloo SE, Metzger D, et al. Validation of diabetes case definitions using administrative claims data. Diabet Med. 2011;28(4):424-27.

13. Buysman E, Conner C, Aagren M, Bouchard J, Liu F. Adherence and persistence to a regimen of basal insulin in a pre-filled pen compared to vial/ syringe in insulin-naïve patients with type 2 diabetes. Curr Med Res Opin. 2011;27(9):1709-17.

14. Goraya TY, Leibson CL, Palumbo PJ, et al. Coronary atherosclerosis in diabetes mellitus: a population-based autopsy study. J Am Coll Cardiol. 2002;40(5):946-53.

15. Malmberg K, Yusuf S, Gerstein HC, et al. Impact of diabetes on longterm prognosis in patients with unstable angina and non-Q-wave myocardial infarction: results of the OASIS (Organization to Assess Strategies for Ischemic Syndromes) Registry. Circulation. 2000;102(9):1014-19.

16. James S, Angiolillo DJ, Cornel JH, et al. Ticagrelor vs. clopidogrel in patients with acute coronary syndromes and diabetes: a substudy from the PLATelet inhibition and patient Outcomes (PLATO) trial. Eur Heart J. 2010;31(24):3006-16.

17. Norhammar A, Lagerqvist B, Saleh N. Long-term mortality after PCI in patients with diabetes mellitus: results from the Swedish Coronary Angiography and Angioplasty Registry. EuroIntervention. 2010;5:891-97. 18. Amsterdam EA, Wenger NK, Brindis RG, et al. 2014 AHA/ACC guideline for the management of patients with non-ST-elevation acute coronary syndrome: a report of the American College of Cardiology/ American Heart Association Task Force on Practice Guidelines. Circulation. 2014;130(25):2354-94.

19. American Diabetes Association. Standards of medical care in diabetes-2013. Diabetes Care. 2013;36(Suppl 1):S11-66.

20. Wiviott SD, Braunwald E, Angiolillo DJ, et al. Greater clinical benefit of more intensive oral antiplatelet therapy with prasugrel in patients with diabetes mellitus in the trial to assess improvement in therapeutic outcomes by optimizing platelet inhibition with prasugrel-Thrombolysis in Myocardial Infarction 38. Circulation. 2008;118(16):1626-36.

21. Zhu B, Zhao Z, McCollam P, et al. Factors associated with clopidogrel use, adherence, and persistence in patients with acute coronary syndromes undergoing percutaneous coronary intervention. Curr Med Res Opin. 2011;27(3):633-41.

22. Cuisset T, Quilici J, Fugon L, et al. Non-adherence to aspirin in patients undergoing coronary stenting: negative impact of comorbid conditions and implications for clinical management. Arch Cardiovasc Dis. 2011;104(5):306-12. 